\title{
Bemerkungen zur deutschen Ausgabe
}

Inorganic Chemistry von James E. Huheey ist in den Vereinigten Staaten eines der beliebtesten und am weitesten verbreiteten Lehrbücher der anorganischen Chemie. Das ungewöhnliche Konzept - den Studenten den Stoff unabhängig von einer am Periodensystem orientierten Systematik unter verschiedenartigen Gesichtspunkten am Leitfaden Principles of Structure and Reactivity nahezubringen und sie an der Entwicklung der Forschung teilhaben zu lassen - hat das Buch auch an deutschen Universitäten zu einem im Unterricht sehr gern und viel benutzten Lehrbuch gemacht. Eine deutsche Ausgabe erschien daher wünschenswert, um den deutschsprachigen Studenten das Erarbeiten des Stoffes, das Verständnis für Zusammenhänge und noch offene Fragen sowie die Einsicht in Probleme und Problematik der chemischen Forschung zu erleichtern und ihnen zu ermöglichen, ungehemmt von Sprachschwierigkeiten aus diesem unkonventionellen Lehrbuch den größtmöglichen Nutzen zu ziehen und die von dem Autor apostrophierte Faszination des Gebietes Anorganische Chemie selbst zu empfinden.

Die vorliegende Ausgabe basiert auf der Übersetzung der zweiten amerikanischen Auflage, die Bertold Reuter noch während seiner Krankheit, unmittelbar vor seinem viel zu frühen Tode als seine letzte Arbeit abgeschlossen hatte und die dann nach der inzwischen erschienenen veränderten dritten Auflage umgearbeitet wurde. Sie folgt im wesentlichen, bis auf einige wenige Änderungen, dem Originaltext. Sonstige ergänzende oder erläuternde Anmerkungen der Übersetzer sind als Fußnoten $(\diamond)$ angegeben. Den Kollegen, die zur Klärung einzelner Punkte hilfreichen Rat gegeben haben, sei dafür auch an dieser Stelle sehr herzlich gedankt.

Um Abbildungen und Tabellen möglichst unverändert aus der amerikanischen Auflage übernehmen zu können, ist auf Wunsch des Verlages der bei Dezimalzahlen im angelsächsischen Schrifttum übliche Punkt beibehalten worden. Fehler in einer Reihe von Abbildungen und Tabellen konnten berichtigt, etliche neue Abbildungen hinzugefügt und einige nach Erscheinen der amerikanischen Auflage veröffentlichte Forschungsergebnisse im Text berücksichtigt werden. Besonderes Augenmerk wurde auf die konsequente Formulierung von Größengleichungen gerichtet. Die Angabe von Größen erfolgt (fast) ausschließlich in SI-Einheiten.

Bei russischen Eigennamen wird die Transliteration des amerikanischen Textes übernommen. Lediglich die nach der deutschen Veröffentlichung zu zitierende Schreibweise des Autornamens Mendelejeff (statt Mendeleev) wird so in der gesamten Übersetzung benutzt.

Die Abkürzung der Zeitschriftentitel folgt dem Chemical Abstracts Service Source Index (CASSI). Da der Text sehr umfangreiche Literaturangaben bringt und nicht nur Originalarbeiten, sondern auch Lehrbücher sowie zusammenfassende Werke und Übersichtsreferate zitiert, wird in der Übersetzung auf ein gesondertes Literaturverzeichnis im Anhang verzichtet. Um dem Benutzer jedoch den Zugang zu der in dem Buch enthaltenen Stoffchemie und der Fülle der verstreuten Hinweise zu erleichtern, ist das Sachverzeichnis gegenüber dem Original erheblich erweitert worden. 
Vielleicht sind noch einige Fehler des Originals erhalten geblieben - mit Sicherheit sind aber etliche Fehler durch Übersetzung und Druck hinzugekommen und trotz sorgfältigen Suchens übersehen worden. Allen kritischen Lesern, die auf derartige Fehler aufmerksam machen, gebührt im voraus Dank für ihre Mühe.

Schließlich gebührt dem Verlag Walter de Gruyter Dank für das Eingehen auf Änderungswünsche und vor allem für die Geduld gegenüber den mehrfach eingetretenen Verzögerungen.

Berlin, im November 1987

Brigitte Sarry 